\title{
A Novel Insight into the Oxidoreductase Activity of Helicobacter pylori HP0231 Protein
}

\author{
Paula Roszczenko ${ }^{1,2}$, Katarzyna A. Radomska ${ }^{1}$, Ewa Wywial ${ }^{3}$, Jean-Francois Collet ${ }^{4,5,6}$, \\ Elzbieta K. Jagusztyn-Krynicka ${ }^{1 *}$
}

1 Department of Bacterial Genetics, Institute of Microbiology, the University of Warsaw, Warsaw, Poland, 2 College of Inter-Faculty Individual Studies in Mathematics and Natural Sciences, the University of Warsaw, Warsaw, Poland, $\mathbf{3}$ Laboratory of Bioinformatics and Protein Engineering, International Institute of Molecular and Cell Biology, Warsaw, Poland, 4 WELBIO (Walloon Excellence in Life Sciences and Biotechnology), Université Catholique de Louvain, Brussels, Belgium, 5 de Duve Institute, Université Catholique de Louvain, Brussels, Belgium, 6 Brussels Center for Redox Biology, Brussels, Belgium

\begin{abstract}
Background: The formation of a disulfide bond between two cysteine residues stabilizes protein structure. Although we now have a good understanding of the Escherichia coli disulfide formation system, the machineries at work in other bacteria, including pathogens, are poorly characterized. Thus, the objective of this work was to improve our understanding of the disulfide formation machinery of Helicobacter pylori, a leading cause of ulcers and a risk factor for stomach cancer worldwide.

Methods and Results: The protein HP0231 from H. pylori, a structural counterpart of E. coli DsbG, is the focus of this research. Its function was clarified by using a combination of biochemical, microbiological and genetic approaches. In particular, we determined the biochemical properties of HP0231 as well as its redox state in $\mathrm{H}$. pylori cells.
\end{abstract}

Conclusion: Altogether our results show that HP0231 is an oxidoreductase that catalyzes disulfide bond formation in the periplasm. We propose to call it HpDsbA.

\begin{abstract}
Citation: Roszczenko P, Radomska KA, Wywial E, Collet J-F, Jagusztyn-Krynicka EK (2012) A Novel Insight into the Oxidoreductase Activity of Helicobacter pylori HP0231 Protein. PLoS ONE 7(10): e46563. doi:10.1371/journal.pone.0046563

Editor: Yoshio Yamaoka, Veterans Affairs Medical Center (111D), United States of America

Received June 17, 2012; Accepted August 31, 2012; Published October 3, 2012

Copyright: (C) 2012 Roszczenko et al. This is an open-access article distributed under the terms of the Creative Commons Attribution License, which permits unrestricted use, distribution, and reproduction in any medium, provided the original author and source are credited.

Funding: The electron microscopy studies were performed in the Laboratory of Electron Microscopy, Nencki Institute of Experimental Biology, Warsaw, Poland by using electron microscope JEM1400 (JEOL Co., Japan, 2008) equipped with an energy-dispersive full range X-ray microanalysis system (EDS INCA Energy TEM Oxford Instruments, Great Britain), a tomographic holder equipment and a high resolution digital camera (CCD MORADA, SiSOlympus, Germany). The above mentioned equipment was installed within the project sponsored by the European Union Structural Funds: Centre of Advanced Technology BIM - Equipment purchase for the Laboratory of Biological and Medical Imaging. The funders had no role in study design, data collection and analysis, decision to publish, or preparation of the manuscript.
\end{abstract}

Competing Interests: The authors have declared that no competing interests exist.

*E-mail: kjkryn@biol.uw.edu.pl

\section{Introduction}

Disulfide bond formation is a post-translational modification, which stabilizes the structure of many extracytoplasmic proteins. In bacteria, disulfides are introduced into proteins secreted to the periplasm by proteins from the Dsb family. In Escherichia coli $(\mathrm{Ec})$, the disulfide bond formation machinery involves two complementary pathways: the oxidation pathway, with EcDsbA and EcDsbB, and the isomerization/reduction pathway, with EcDsbC and EcDsbD [1,2]. The first pathway is responsible for the formation of disulfide bonds in newly synthesized proteins, while the second catalyzes the rearrangement of improperly introduced disulfides [3].

EcDsbA is a $21 \mathrm{kDa}$ monomeric protein from the thioredoxin family, which donates the disulfide bond present in its active site to reduced protein substrates. This reaction depends on the presence of two highly conserved motifs: a CXXC (CPHC) active site motif and the so-called cis-proline loop [4]. Both motifs are distant in linear sequence but close in the three-dimensional structure. EcDsbA is maintained in an oxidized form in vivo due to the action of $\mathrm{EcDsbB}$, a $20 \mathrm{kDa}$ membrane protein that generates disulfides de novo from quinone reduction. Thus, disulfide bond formation is connected to the electron transport chain.

EcDsbA does not have a rearranging activity and can introduce non-native disulfides into proteins with multiple cysteine residues. In $E$. coli, the periplasmic protein disulfide isomerase EcDsbC is responsible for the rearrangement of those incorrect disulfides. $\mathrm{EcDsbC}$ is a $23.3 \mathrm{kDa}$ protein that folds into a $\mathrm{V}$-shaped homodimer [5]. Each monomer of DsbC consists of two domains: a Gterminal catalytic domain with a thioredoxin fold and a $\mathrm{N}$ terminal dimerization domain that also plays a role in substrate binding.

In E. coli, the periplasm contains an additional oxidoreductase, $\mathrm{EcDsbG}$, which is a structural homologue of EcDsbC [6]. EcDsbG has recently been shown to control the levels of cysteine sulfenylation in the periplasm [7].

EcDsbC and EcDsbG both have catalytic CXXC motifs that are kept in the reduced state by the integral membrane protein EcDsbD. EcDsbD, which catalyzes the transfer of electrons from the cytoplasmic thioredoxin system to the periplasm, consists of eight transmembrane segments ( $\beta$ domain), a N-terminal $(\alpha$ 
Table 1. Bacterial strains and plasmids used in this study.

\begin{tabular}{|c|c|c|}
\hline Name & Relevant characteristics & Source/Ref. \\
\hline \multicolumn{3}{|c|}{ Strains: Helicobacter pylori } \\
\hline 26696 & H. pylori wild-type & ATCC \\
\hline N6 & H. pylori wild-type & [19] \\
\hline PR305 & N6 dsbl::aph & This study \\
\hline PR336 & $d s b l^{+}$in trans complementant of $d s b l:: a p h$ & This study \\
\hline PR378 & N6 hp0231::cat & This study \\
\hline PR397 & hp0231 ${ }^{+}$in trans complementant of hp0231::cat & This study \\
\hline \multicolumn{3}{|l|}{ Escherichia coli } \\
\hline TG1 & 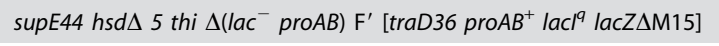 & [21] \\
\hline BL21 & 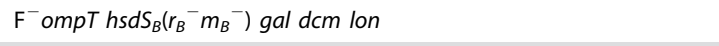 & Novagen \\
\hline JCB816 & MC1000 phoR $\lambda 102$ & [44] \\
\hline JCB817 & JCB816 dsbA:kan1 & [44] \\
\hline JCB818 & JCB 816 dsbA::kan1 dsbB::kan & [44] \\
\hline JFC383 & JCB816 dsbC::kan & JFC collection \\
\hline JFC571 & JFC383 pHEL2 & This study \\
\hline JFC572 & JFC383 pUWM500 & This study \\
\hline PR501 & JCB 817 pHEL2 & This study \\
\hline PR503 & JCB 817 pUWM500 & This study \\
\hline PR521 & JCB 818 pHEL2 & This study \\
\hline PR522 & JCB 818 pUWM500 & This study \\
\hline \multicolumn{3}{|l|}{ Plasmids: } \\
\hline pET22b & $A p^{r}$, IPTG inducible & Novagen \\
\hline pET28a & $\mathrm{Km}^{r}$, IPTG inducible & Novagen \\
\hline pGEM-T Easy & $A p^{r} ; \operatorname{LacZ} \alpha$ & Promega \\
\hline pHEL2 & $\mathrm{Cm}^{r}$ E. coli/H. pylori shuttle vector & [45] \\
\hline pHEL3 & $\mathrm{Km}^{\mathrm{r}}$ E. coli/H. pylori shuttle vector & [45] \\
\hline pRY109 & $\mathrm{Cm}^{r}$ & [41] \\
\hline pUWM305 & pBluescript II SK/dsbl::aph & [10] \\
\hline pUWM336 & $d s b I^{+}$in $\mathrm{pHEL} 2$ & [10] \\
\hline pUWM378 & pGEM-T Easy/hp0231::cat & This study \\
\hline pUWM382 & $\mathrm{Ap}^{\mathrm{r}} \mathrm{hp} 0231^{+}$in $\mathrm{pET} 22 \mathrm{~b}$ & This study \\
\hline pUWM589 & hp0231 in pGEM-T Easy & This study \\
\hline pUWM397 & hp0231 $1^{+}$in pHEL3 & This study \\
\hline pUWM500 & $h p 0231^{+}$in pHEL2 & This study \\
\hline pUWM525 & $\mathrm{Km}^{r} \mathrm{hp} 0231^{+}$in pET28a & This study \\
\hline
\end{tabular}

domain) and a C-terminal domain ( $\gamma$ domain). Both the $\mathrm{N}$ - and $\mathrm{C}$ terminal domains are present in the periplasm [8].

In contrast to the $E$. coli system which has been extensively studied, the $H$. pylori disulfide bond formation system has been poorly characterized although this system plays an important role in the colonization process [9]. Previous research has revealed that the H. pylori genome encodes a homologue of EcDsbB (HpDsbI) [10] but no close homologue of EcDsbA. There are also two additional thioredoxin-like CXXC-containing periplasmic proteins, HP0231 and HP0377. These proteins share only 12\% and $24 \%$ identity with EcDsbA, respectively, and their function is unknown [11].

$H$. pylori infection induces a strong inflammatory response in the stomach. This response is manifested by generation of large amounts of reactive oxygen species/reactive nitrogen species
(ROS/RNS) produced by infiltrating host cells. The long-term exposure to ROS contributes to the development of cancerous gastric cells $[12,13]$. Thus, the pathogen has evolved a defense system involving several antioxidant enzymes, such as superoxide dismutase, catalase or alkyl hyperoxide reductase [14]. Additionally, oxidoreductases, specifically disulfides reductases, play a major role in the antioxidant defense mechanism and in the maintenance of intracellular redox balance. Some of them can even act in the oxidative environment of the cell envelope [15]. Because the $H$. pylori is equipped with only one intracellular disulfide reducing system, the thioredoxin system, and lacks the glutaredoxin/glutathione system and because the $H$. pylori Dsb system is still poorly understood molecular characterization of the Dsb proteins may also help to expand our knowledge about the mechanism used by $H$. pylori to combat oxidative stress. 
We decided to genetically and biochemically study HP0231, a protein which has been selected as a component of a subunit antiHelicobacter vaccine [16,17], to shed light on its function. To achieve this goal, we generated a $H$. pylori hp0231 mutant and subsequently carried out its phenotypic characterization. We also examined whether HP0231 is able to complement an E. coli $d s b A$ mutant and we biochemically characterized the protein. Moreover, we determined the redox state of HP0231 in wild-type and a $d s b I$ mutant strain of $H$. pylori.

\section{Materials and Methods}

\section{Ethics Statement}

All studies involving animals were performed in accordance with ethical standards, after approval from the Local Ethic Committee No. 1 Warsaw, Poland 966/2009.

\section{Bacterial strains, primers, plasmids, media and growth conditions}

Bacterial strains, plasmids and primers used in this study are listed in Tables 1 and 2. Two H. pylori strains (26695 and N6) were used in this study. Although the sequence of the H. pylori 26695 genome is completed [18], this strain is inconvenient for genetic manipulation. H. pylori $\mathrm{N} 6$, originally isolated from a patient with gastritis, is useful for complementation experiments and highly motile [19]. Both H. pylori strains were grown on blood agar base 2 (BA) plates (Merck) supplemented with 10\% horse blood and an antibiotic mixture consisting of vancomycin (final concentration, $\left.12.5 \mu \mathrm{g} \mathrm{ml}^{-1}\right)$, polymyxin B $\left(1.25 \mu \mathrm{g} \mathrm{ml}^{-1}\right)$, trimethoprim $\left(6.25 \mu \mathrm{g} \mathrm{ml}^{-1}\right)$ and amphotericin $\mathrm{B}\left(2.5 \mu \mathrm{g} \mathrm{ml}{ }^{-1}\right)$ at $37^{\circ} \mathrm{C}$ under microaerobic conditions by using CampyGen (Oxoid). Liquid cultures of $H$. pylori were grown in Brain Heart Infusion (BHI) broth supplemented with $10 \%$ fetal bovine serum (FBS). For the selection of $H$. pylori mutated or complemented strains, kanamycin $\left(25 \mu \mathrm{g} \mathrm{ml}^{-1}\right)$ or/and chloramphenicol $\left(10 \mu \mathrm{g} \mathrm{ml}^{-1}\right)$ were added to the growth media.

The E. coli strain TG1 was used as a host for the construction and preparation of recombinant plasmids. The E. coli strain BL21 was used to overexpress pUWM382 and pUWM525. The E. coli strains JCB817 and JCB818 were employed for complementation experiments of E. coli $d s b A$ mutant by HP0231. E. coli strains were grown at $37^{\circ} \mathrm{C}$ on solid or liquid Luria-Bertani (LB) medium or on M63 minimal medium [20]. When needed, media were supplemented with antibiotics at the following concentrations: $100 \mu \mathrm{g} \mathrm{ml}^{-1}$ of ampicillin, $30 \mu \mathrm{g} \mathrm{ml}^{-1}$ of kanamycin and $20 \mu \mathrm{g} \mathrm{ml}^{-1}$ of chloramphenicol.

\section{General DNA manipulations}

Standard DNA manipulations were carried out as described earlier [21] or according to the manufacturer's instructions (A\&A Biotechnology). Polymerase chain reactions (PCR) were performed with PrimeStar HS DNA Polymerase (Takara) or HotStar HiFidelity Polymerase (Qiagen) under standard conditions. Synthetic oligonucleotides synthesis and DNA sequencing were performed by Genomed S.A.,Warsaw, Poland.

\section{Natural transformation of $H$. pylori}

The naturally competent $H$. pylori $\mathrm{N} 6$ was grown on BA plates for $24 \mathrm{~h}$. Subsequently, bacteria were plated onto fresh plates for $5 \mathrm{~h}$. Then $0.5-1 \mu \mathrm{g}$ of plasmid DNA was added and plates were incubated for $22 \mathrm{~h}$. Afterwards bacteria were transferred onto a plate supplemented with chloramphenicol or kanamycin and transformants were grown for 5 days.

\section{Allelic exchange mutagenesis of $d s b$ genes in $H$. pylori}

To inactivate $h p 0231$, the recombinant vector was constructed by a two-step PCR method [22]. Briefly, primers Cat1-Cat2, were used to amplify the cat gene from pRY109. The upstream and downstream regions of the $h p 0231$ gene were amplified from $H$. pylori 26695 genomic DNA using two pairs of primers specific for hp0231 and its flanking regions, 1HP231Xh-2HP231 and 3HP231-4HP231, respectively. The 2HP231 and 3HP231 primers contained 5' leader nucleotide sequences complementary to Cat 1 and Cat2, respectively. Each PCR product was purified by GelOut extraction kit (A\&A Biotechnology). Next a mixture of three purified products (in equal amounts) was used as a template in a single PCR reaction, using primers: $1 \mathrm{HP} 231 \mathrm{Xh}-4 \mathrm{HP} 231$. Subsequently, the resulted PCR product, containing the cat gene inserted between two $h p 0231$ arms in the same transcriptional orientation as the $h p 0231$ gene, was purified and cloned into pGEM-T Easy generating a suicide plasmid pUWM378. Sequence analyses confirmed the correct construction of pUWM378

Table 2. Primers used in this study.

\begin{tabular}{|c|c|c|}
\hline Name & Sequence $5^{\prime}-3^{\prime}$ & Orientation/Restriction site \\
\hline 1HP231Xh & GTGCTCGAGGCCTGCTCTTCATCAATAACTTTAG & Forward/Xhol \\
\hline $2 \mathrm{HP} 231$ & ATCCACTTTTCAATCTATATCCAACACACTCGCTCTTAATATC & Reverse \\
\hline 3HP231 & CCCAGTTTGTCGCACTGATAATTGAATCTGGCGTGATTAAGG & Forward \\
\hline 4HP231 & TAGGATCCCTTGTGGGGATTTGTAGGTC & Reverse/BamHI \\
\hline Cat1 & GATATAGATTGAAAAGTGGAT & Forward \\
\hline Cat2 & TTATCAGTGCGACAAACTGGG & Reverse \\
\hline 5aHP231 & AAGCGATGATGTGCAATTAG & Forward \\
\hline 5bHP231 & GAGCGTTAATATCGTATTGG & Reverse \\
\hline 231 expl & GAGGCCATGGCTAATGACAAACGGATGCAG & Forward/Ncol \\
\hline 231 expll & GTGCTCGAGTGCCTTATAATGGTATAAGAA & Reverse/Xhol \\
\hline HP232F & AGCGCGTTAGTGATGGTGAG & Forward \\
\hline HP232R-RT & GCTGGTAATTAGGGTTATTG & Reverse \\
\hline
\end{tabular}

Restriction recognition sites introduced for cloning purposes are underlined. All primers were designed on the basis of $H$. pylori 26695 genome nucleotide sequence. doi:10.1371/journal.pone.0046563.t002 
and the recombinant plasmid was introduced into $H$. pylori $\mathrm{N} 6$ by natural transformation.

A $h p 0231::$ cat mutant was obtained by a double crossing over by using pUWM378, and verified by PCR analysis using a pair of primers 5aHP231-5bHP231. The lack of HP0231 in the proteome of the mutated strain was confirmed by Western blot analysis using specific rabbit polyclonal anti-HP0231 antibodies.

Inactivation of $d s b I$ (hp0595) was carried out by transforming a $H$. pylori N6 wild type strain with the previously constructed pUWM305 plasmid [10]. Transformants were grown for 5 days on selective plates supplemented with kanamycin. Allelic exchange in mutagenesis resulting in a $d s b I::$ aph mutant was confirmed by PCR. Additionally, the lack of DsbI in the proteome of the mutated strain was verified by Western blot analysis using specific rabbit polyclonal anti-DsbI antibodies.

\section{Construction of $h p 0231^{+}$plasmids for complementation experiments}

To analyze the complementation of the hp0231 mutation in $H$. pylori $\mathrm{N} 6$ and the $d s b A$ mutant in $E$. coli JCB 816 by HP0231, two recombinant plasmids were constructed based on shuttle $E$. coli/H. pylori plasmids: pHEL3 and pHEL2. Because the H. pylori hp0231 mutant and the E. coli $d s b A$ mutant carry different genes responsible for antibiotic resistance we used two different plasmids as a starting point of these experiments. However, both plasmids had the same replication system, thus were present in similar copy number. The $h p 0231$ coding nucleotide sequence with its own promoter was amplified from $H$. pylori 26695 genomic DNA, using a pair of primers: 1HP0231Xh-4HP0231. The purified PCR products as well as the shuttle plasmids were digested with XhoI/ BamHI and ligated together to form pUWM397 and pUWM500. Correct construction of pUWM397 and pUWM500 was confirmed by sequencing. Next, pUWM397 was introduced into $H$. pylori N6 lacking hp0231 and pUWM500 was used for the E. coli DsbA complementation tests.

\section{Construction of $d s b I^{+}$plasmids for complementation experiments}

The recombinant plasmid pUWM336 was introduced into $H$. pylori PR 305 by natural transformation (described above) in order to complement a mutation in the $d s b I$ gene.

\section{RT-PCR}

RT-PGR reactions were carried out as described earlier [23]. Total RNAs were extracted from H. pylori N6 and H. pylori PR378 using the standard TRIzol procedure (Invitrogen). After DNase I treatment, RNA was reverse-transcribed using Omniscript Reverse Transcriptase (Qiagen) and a primer HP232R-RT. PCR reactions without reverse transcriptase were used to determine whether RNA was free of DNA contamination. PCR reactions performed on cDNA were carried out with a pair of primers: HP232F-HP232R-RT.

Standard protein manipulations were carried out as described earlier [21].

\section{Overexpression and purification of HP0231}

A HP0231 expression vector was constructed by amplifying the region encoding the mature HP0231 protein (without the signal sequence, amino acids 1-24), from the chromosome of $H$. pylori 26695 with primers $231 \operatorname{expI}$ and $231 \mathrm{expII}$. For cloning the insert into pET22b and pET28a, NcoI and XhoI restriction enzymes were used to yield plasmids, pUWM382 and pUWM525, respectively. Both plasmids carried $\mathrm{HP} 0231-\mathrm{His}_{6}$ translation fusion. The periplasm-located HP0231 was overexpressed from pUWM382 by autoinduction [24] and then purified by affinity chromatography, dialyzed against PBS (Sigma) and later used for rabbit immunization (Animal Facility, Faculty of Biology, University of Warsaw). The anti-HP0231 rabbit serum was specific and recognized native HP0231, as was verified by Western blot analysis.

For the biochemical experiments the protein was expressed and purified from $E$. coli BL21 harboring pUWM525. Expression was induced with $1 \mathrm{mM}$ IPTG at $\mathrm{A}_{600} \sim 0.6$. After $4 \mathrm{~h}$, cultures were centrifuged and the cell pellet was suspended in $50 \mathrm{mM}$ sodium phosphate, $\mathrm{pH} 8.0,300 \mathrm{mM}$ NaCl. Cells were disrupted by 2 passes through a French Press Cell at 1200 PSI. The cell lysate was centrifuged and the resulting supernatant was applied onto HisTrap column (Qiagen). The protein was eluted with an imidazole gradient. Fractions containing HP0231 were pooled and loaded onto a PD-10 column (GE Healthcare) equilibrated with $50 \mathrm{mM}$ sodium phosphate, $\mathrm{pH}$ 8.0, $150 \mathrm{mM}$ NaCl. To obtained higher purity HP0231 fractions were pooled and diluted 10-fold with $20 \mathrm{mM}$ Tris, pH 8.0 and later loaded onto Sepharose column equilibrated with the same buffer. The protein was eluted with $\mathrm{NaCl}$ gradient. Fractions containing HP0231 were pooled, concentrated and loaded onto a PD-10 column equilibrated with $50 \mathrm{mM}$ sodium phosphate, $\mathrm{pH}$ 8.0, $150 \mathrm{mM} \mathrm{NaCl}$.

\section{In vivo redox state of HP0231}

The redox state of HP0231 was visualized by alkylating the free cysteine residues using 4-acetamido-4'-maleimidylstilbene-2,2' disulfonic acid (AMS, Invitrogen). This agent can only modify covalently free thiols, resulting in a molecular mass increase of 490 Da. Briefly, bacteria were harvested from BA plates after 24 or $48 \mathrm{~h}$ of incubation under microaerobic conditions. Samples were standardized using $\mathrm{OD}_{600}$ of the culture and ice-cold trichloroacetic acid (TCA, final concentration $10 \% \mathrm{v} / \mathrm{v}$ ) was immediately added directly to the culture. Whole-cell proteins were precipitated and collected by centrifugation, then washed with ice-cold acetone and dissolved in $50 \mathrm{mM}$ Tris- $\mathrm{HCl}(\mathrm{pH} 7.5)$, $10 \mathrm{mM}$ EDTA, $0.1 \%$ SDS containing $20 \mathrm{mM}$ AMS by agitation for $45 \mathrm{~min}$ at $37^{\circ} \mathrm{C}$. The proteins in non-reducing Laemmli buffer were resolved by $18 \%$ SDS-PAGE without reducing agent. HP0231 was then detected by immunoblot analysis using an anti-HP0231 antibody.

As controls, we used samples previously treated with $100 \mathrm{mM}$ DTT for $30 \mathrm{~min}$ at room temperature before precipitation of the proteins with TCA.

\section{Insulin reduction assay}

The ability of HP0231 and EcDsbA to catalyze the reduction of insulin in the presence of DTT was determined as previously described [25].

\section{Determination of the redox potential of HP0231}

The redox potential of HP0231 was fluorimetrically determined at $\mathrm{pH} 7$ at room temperature from the equilibrium constant with glutathione as previously described [25]. Briefly, experiments were performed at room temperature in a buffer containing $100 \mathrm{mM}$ sodium phosphate (pH 7.0) and 1 mM EDTA. HP0231 (1 $\mu \mathrm{M})$ was incubated for $12 \mathrm{~h}$ in the presence of $0.1 \mathrm{mM}$ GSSG and varying concentrations of GSH $(0.4 \mu \mathrm{M}$ to $8 \mathrm{mM})$. For different GSH/GSSG ratios, the fraction of oxidized and reduced HP0231 was determined from the increase of the tryptophan fluorescence upon reduction. The fraction $(\mathrm{R})$ of each of the reduced proteins at equilibrium with glutathione was measured using specific fluorescence of the protein at $330 \mathrm{~nm}$ (excitation $295 \mathrm{~nm}$ ). The 
equilibrium constant $\left(\mathrm{K}_{\mathrm{eq}}\right)$ was determined by fitting the obtained data to the equation:

$$
\mathrm{R}=\left([\mathrm{GSH}]^{2} /[\mathrm{GSSG}]\right) /\left(\mathrm{K}_{\mathrm{eq}}+[\mathrm{GSH}]^{2} /[\mathrm{GSSG}]\right)
$$

The redox potential was calculated using the Nernst equation. The value of $-240 \mathrm{mV}$ was used as a standard potential for glutathione.

$$
\mathrm{E}_{\mathrm{HP} 0231}^{\circ \prime}=\mathrm{E}_{\mathrm{GSH} / \mathrm{GSSG}}^{\circ}(\mathrm{RT} / 2 \mathrm{~F}) \ln \mathrm{K}_{\mathrm{eq}}
$$

\section{DTT sensitivity assay}

DTT sensitivity experiments were performed as previously described [26] with some modifications concerning $H$. pylori growth requirements. Briefly, a freshly prepared $1 \mathrm{M}$ DTT (Sigma) stock solution was dissolved in molten BA supplemented with $10 \%$ horse blood to the final concentration of $8 \mathrm{mM}$ DTT (for $H$. pylori assay) or in molten LB agar to the final concentration of $12 \mathrm{mM}$ DTT (for $E$. coli assay). The amount of DTT used completely inhibits the growth of the E. coli strain JCB817, but not the wild type parental strain. The DTT agar plates were used within $30 \mathrm{~min}$ of pouring to prevent oxidation of DTT by air. Exponentially growing cultures were serially diluted 10-fold, and $7 \mu \mathrm{l}$ aliquots were spotted on the plates. The growth was observed after $48-72 \mathrm{~h}$ of incubation for the $H$. pylori assay and after overnight incubation at $37^{\circ} \mathrm{C}$ for the E. coli assay. The experiments were conducted in triplicate.

\section{Motility assays}

H. pylori strains were grown in BHI supplemented with $10 \%$ heat-inactivated FCS until mid-log phase. Next, cells were inoculated on Mueller-Hinton $(\mathrm{MH})$ soft agar plates containing $0.35 \%(\mathrm{w} / \mathrm{v})$ agar and $10 \%(\mathrm{v} / \mathrm{v})$ FCS with a sterile toothpick and incubated for 4 to 5 days at $37^{\circ} \mathrm{C}$ under microaerobic conditions.

E. coli cells were grown in liquid culture in LB broth until the $\mathrm{OD}_{600}$ value was close to 1 . Then bacteria were inoculated on $\mathrm{LB}$ soft agar plates containing $0.35 \%(\mathrm{w} / \mathrm{v})$ agar with a sterile toothpick and incubated for $18 \mathrm{~h}$ at $30^{\circ} \mathrm{C}$.

\section{Transmission electron microscopy}

Bacteria were grown on BA plates for $48 \mathrm{~h}$ under microaerobic conditions, then washed three times with PBS and suspended in $1.5 \%(\mathrm{v} / \mathrm{v})$ glutaraldehyde. Subsequently, the bacteria were transferred to a 400-mesh carbon - coated copper grid by floating the grid, coated side down on a drop of $H$. pylori culture fluid for $30 \mathrm{~s}$. Next, the bacteria were negatively stained by floating the grid on a drop of $1 \%(\mathrm{w} / \mathrm{v})$ phosphotungstic acid solution for $20 \mathrm{~s}$. The excess of reagent was washed twice by floating the grid on a drop of water for $10 \mathrm{~s}$. The grid was allowed to dry in air. Samples were examined on an electron microscope JEM 1400 (JEOL Co., Japan, 2008).

Measurements of 100 cells/strain were taken manually from TEM images by drawing the central axis of each cell running from pole to pole, in the iTEM 5.0 (Build 1235) Olympus Soft Imaging Solution $\mathrm{GmbH})$.

\section{Alkaline phosphatase (AP) assay}

AP activity was measured as previously described [16] with some modifications. Briefly, E. coli cell cultures were grown at $37^{\circ} \mathrm{C}$ in minimal medium M63 until they reached mid-log phase.
Then, the cells were pelleted by centrifugation, washed twice with Tris-HCl ( $\mathrm{pH}$ 8.0), and resuspended in Tris-HCl ( $\mathrm{pH}$ 8.0). The $\mathrm{OD}_{600}$ was measured spectrophotometrically. Samples $(1 \mathrm{ml})$ were equilibrated for $5 \mathrm{~min}$ in water bath at $28^{\circ} \mathrm{C}$, then $200 \mu \mathrm{l} \mathrm{p}$ nitrophenol phosphate $[0.4 \%(\mathrm{w} / \mathrm{v})$ in Tris-HCl $(\mathrm{pH} 8.0)]$ was added, and time was recorded. The reaction was allowed to proceed at $28^{\circ} \mathrm{C}$ until the development of yellow color was observed. At this point the reaction was stopped by adding $200 \mu \mathrm{l}$ $1 \mathrm{M} \mathrm{KH}_{2} \mathrm{PO}_{4}$. Samples were centrifuged before the $A_{420}$ and $A_{550}$ measurements.

\section{Copper sensitivity assay}

For copper sensitivity assay bacteria were grown in BHI media supplemented with $8 \mathrm{mM}$ or $10 \mathrm{mM} \mathrm{CuCl} 2$. Strains were grown at $34^{\circ} \mathrm{C}$.

\section{Statistical analysis}

Statistical analyses of variance (ANOVA) and ANOVA specifying the "Welch" option controls for unequal variances followed by the Tukey's post hoc test, as well as the mean and SD calculations were carried out using MS Excel 2007 with SigmaXL.

\section{Results}

\section{Determination of the in vivo redox state of HP0231}

When we started this work, we first performed fold-recognition analyses that suggested that the fold of HP0231 resembles that of EcDsbG. This was confirmed by the recently published structure of HP0231 [27]. In this structure, HP0231 appears as a V-shaped dimeric protein, which is reminiscent of the oxidoreductases that function in the reductive pathways present in the $E$. coli periplasm. However, both the facts that the $H$. pylori genome does not encode a classical monomeric DsbA protein, despite the presence of a $d s b B$-like gene $(h p d s b I)$, and that the catalytic site of HP0231 is identical to that of EcDsbA (both proteins have a CPHC active site) lead us to suspect that HP0231 might be involved in disulfide bond formation rather than in disulfide isomerization.

Thus, to clearly define whether HP0231 functions in the oxidizing or the reducing pathway, we first decided to determine the redox state of that protein in vivo. The in vivo redox state of an oxidoreductase usually reflects its activity in the cell, i.e., proteins that function in the oxidizing pathway, such as EcDsbA, are maintained oxidized in vivo, whereas proteins that function in the reducing pathway are maintained predominantly reduced.

We used the AMS-trapping technique to determine the in vivo redox state of HP0231. We found that HP0231 is present in the oxidized form in wild-type cells, which suggests that HP0231 functions in the oxidizing pathway in $H$. pylori. We then tested whether deletion of $h p d s b I$ affects the redox state of HP0231 (remembering that EcDsbB is the protein that recycles EcDsbA). As shown in Figure 1, the deletion of the hpdsbI results in the accumulation of a noticeable amount of HP0231 in the reduced state. Thus, these data strongly suggest that HP0231, despite its structural resemblance to $\mathrm{EcDsbG}$, functions in the oxidizing pathway in the H. pylori periplasm and that HP0231 is maintained oxidized by HpDsbI.

\section{Phenotypic characterization of HP0231 mutated cells}

To shed more light on HP0231's function, we constructed an isogenic $h p 0231$ knock-out strain by allelic exchange methodology. A recombinant plasmid (based on a vector non-replicating in Helicobacter cells) was used for the mutagenesis. It contained the hp0231 gene disrupted by insertion of a chloramphenicol resistance cassette into the gene coding sequence. The correctness of the 


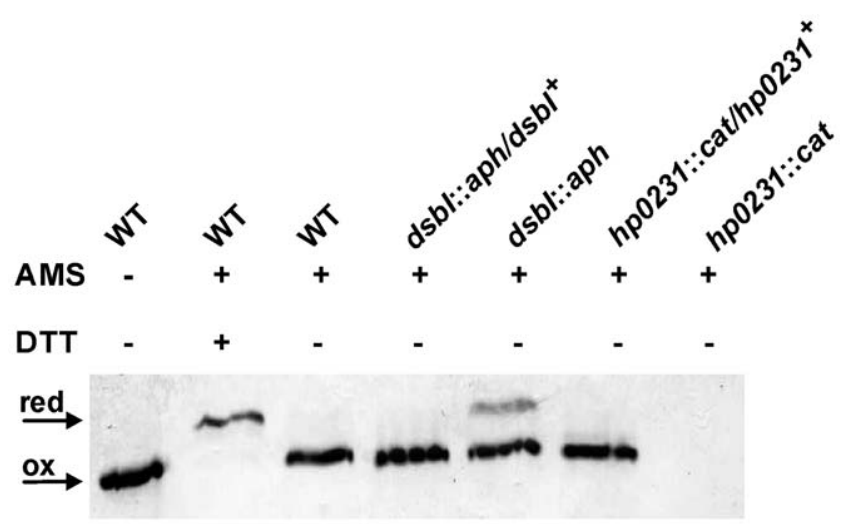

Figure 1. Redox state of HP0231 in WT, mutants: dsbl:aph, hp0231::cat and complemented strains. Bacterial cultures were treated with $10 \%$ TCA, followed by alkylation with AMS. Cellular proteins including the reduced (red; DTT treated, modified with AMS) and the oxidized (ox; non-modified with AMS) controls were separated by $18 \%$ SDS-PAGE under non-reducing conditions, and Western blot analysis using antibodies against HP0231 was performed. Each lane contains proteins isolated from the same amount of bacteria. doi:10.1371/journal.pone.0046563.g001

plasmid construction was confirmed by PCR and DNA sequencing. The expected disruption of the chromosomal hp0231 locus as a result of the double cross-over recombination event was verified by PCR using appropriate pairs of primers. The loss of the wildtype $h p 0231$ gene product was also confirmed by Western blotting of whole-cell proteins with specific anti-HP0231 antibodies (Figure $\mathrm{S} 1)$. We also verified that the antibiotic cassette disrupting the hp0231 gene (introduced in the same transcriptional orientation as the mutated gene) causes no polar effect on the expression of the adjacent gene ( $h p 0232)$ using RT-PCR (Figure S2).

As the E. coli $d s b A$ mutant is sensitive to dithiotreitol (DTT), we first decided to compare the DTT sensitivity of the $h p 0231$ mutant to that of wild type cells. We found that $H$. pylori lacking HP0231 is DTT-sensitive and shows reduced growth compared to the wildtype parental strain (Figure 2).
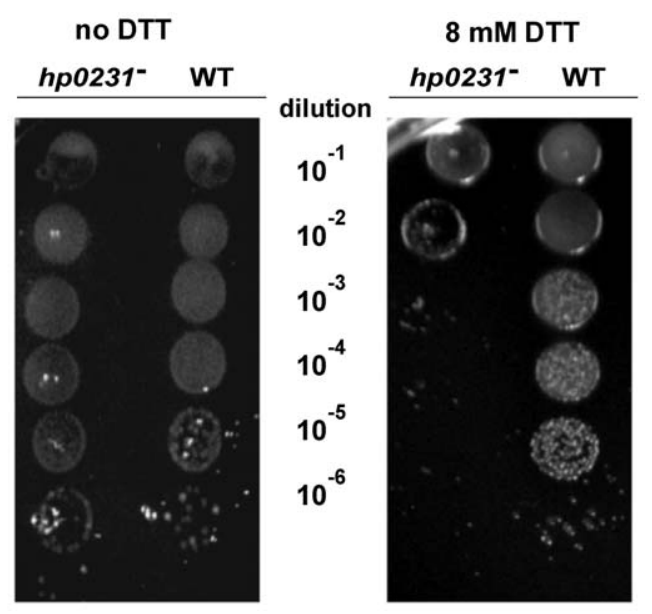

Figure 2. The $H$. pylori N6 hp0231::cat mutant is DTT sensitive. Exponentially growing $H$. pylori wt and $h p 0231:: c a t$ cultures were tenfold serial diluted and spotted on BA plates without (panel $A$ ) or with (panel B) $8 \mathrm{mM} \mathrm{DTT}$, and incubated at $37^{\circ} \mathrm{C}$. The mutant shows reduced growth after 3 days of incubation on plates containing DTT. doi:10.1371/journal.pone.0046563.g002
Bacteria from many genera that contain a mutation in $d s b A$ or $d s b B$ are non-motile, because of their inability to produce functional flagella [28]. As shown in Figure 3, the hp0231 mutant is non-motile. To clarify the reason for the lack of motility, we performed transmission electron microscopy experiments to visualize potential changes in the $H$. pylori flagellar elements and/or in cellular morphology. As shown in Figure 4, we did not observe any defect in the flagella distribution or structure. We observed, however, some changes in the mutant morphology. The hp0231 gene inactivation yields cells either with straight-rod or curved-rod morphology. Additionally, on average, these cells are longer (almost 50\% longer) than the wild-type cells. A wild-type phenotype could be restored by hp0231 complementation in trans.

\section{HP0231 complements an E. coli $d s b A$ mutant}

We then tested the ability of HP0231 to complement the E. coli $d s b A$ mutant. E. coli strains lacking a functional $d s b A$ exhibits a pleiotropic phenotype such as loss of motility, DTT sensitivity and low alkaline phosphatase activity [4]. HP0231 was expressed in the E. coli $d s b A$ mutant to determine the ability of this protein to suppress the defects associated with the lack of disulfide bond formation. Expression of the protein was confirmed by Western blot using a specific rabbit anti-HP0231 antibody (data not shown). We first tested whether the expression of HP0231 in the $d s b A$ mutant leads to increased levels of alkaline phosphatase activity and found that expression of HP0231 restores about 50\% of the PhoA activity measured in the wild-type strain (Figure 5B). Then, in order to assess whether the activity of HP0231 in E. coli is dependent on the presence of $E$. coli DsbB, we introduced a plasmid-encoding HP0231 into E. coli JCB818 cells (dsbA dsbB double mutant). However, we observed that the absence of EcDsbB only slightly decreases the alkaline phosphatase activity.

We also found that the expression of HP0231 in the E. coli $d s b A$ mutant both restores the motility of this strain (Figure 5C) and suppresses the DTT-sensitivity (Figure 5A).

Taken together, our results clearly indicate that HP0231, despite its structural resemblance to EcDsbG, can complement the E. coli $d s b A$ mutant, suggesting that HP0231 is able to catalyze disulfide bond formation in the bacterial periplasm.

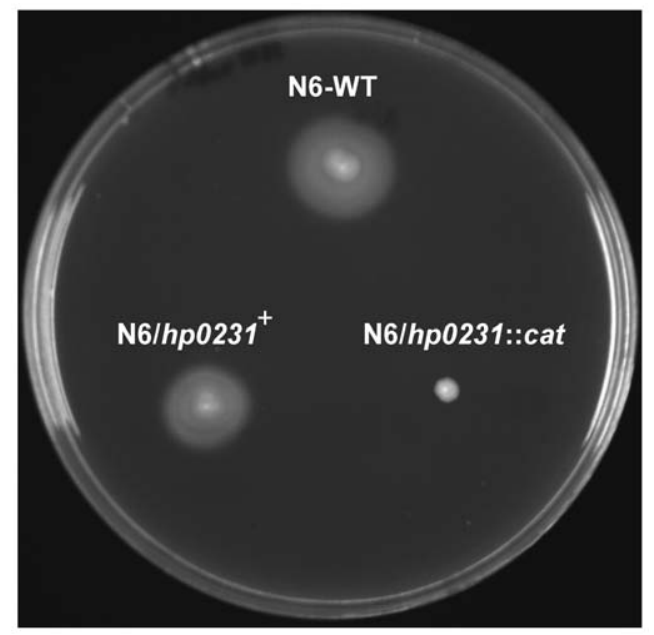

Figure 3. Motility of $H$. pylori N6 strains: wt, hp0231::cat and hp0231::cat complemented in trans by puWM397 (hp0231 $)$. Bacterial motility was monitored after 4 days of incubation on $0.3 \% \mathrm{MH}-$ agar plates containing 10\% FCS. The hp0231 mutant strain is nonmotile.

doi:10.1371/journal.pone.0046563.g003 
A

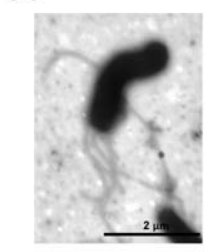

B

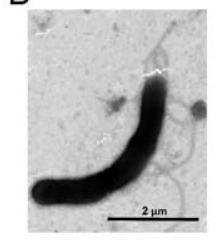

C

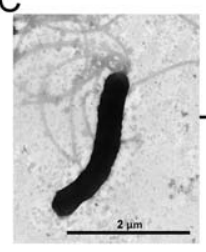

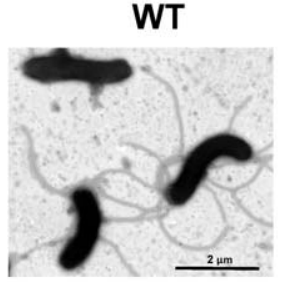

hp0231::cat

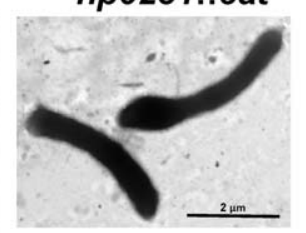

hp0231::cat/hp0231+

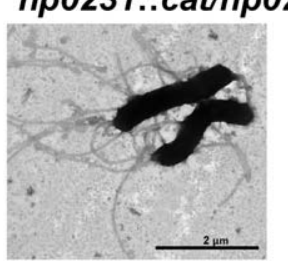

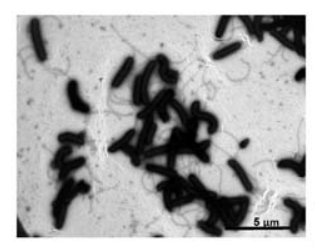
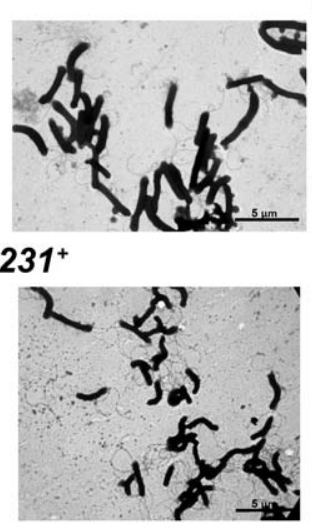

D

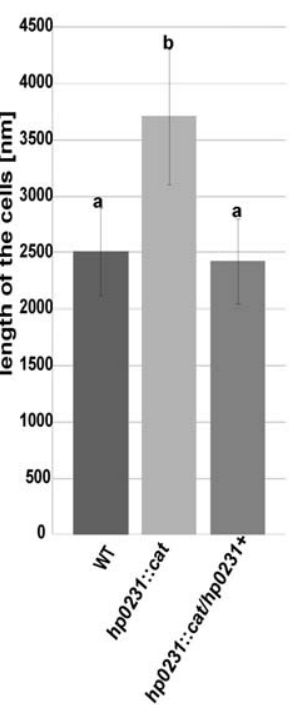

Figure 4. Comparison of H. pylori N6 cells (wt, hp0231::cat and hp0231::cat complemented in trans by puWM397 (pHel2/hp0231) morphology by transmission electron microscopy (TEM). A) WT, B) hp0231::cat and C) hp0231::cat/hp0231 (hp0231::cat complemented in trans by wild type copy of hp0231). D) The diagram illustrates mean lengths and standard deviations of 100 bacterial cells in $\mathrm{nm}$. Error bars with the different letters indicate a significant difference $(p<0.01)$ in length between the $H$. pylori N6 wt cells and the mutant strain, and the mutant and the complementant strain (Welch ANOVA followed by post hoc Tukey's test). doi:10.1371/journal.pone.0046563.g004

\section{HP0231 does not complement an E. coli $d s b C$ mutant}

We also tested whether the expression of HP0231 in the $d s b C$ mutant restores viability characteristic for wild-type cells under copper stress conditions. We found that HP0231 does not complement the $d s b C$ mutation (Figure S3) suggesting that HP0231 is not active in the isomerization pathway.

\section{Biochemical characterization of HP0231}

To characterize the biochemical properties of HP0231, we produced a recombinant protein in $E$. coli. We first determined whether HP0231 is able to catalyze the reduction of insulin by DTT. Insulin contains two intramolecular disulfide bonds that connect the $\mathrm{A}$ and $\mathrm{B}$ chains: reduction of these disulfide bonds causes the precipitation of the $\mathrm{B}$ chain, which can be monitored by following the increase of turbidity at $650 \mathrm{~nm}[29,30]$. The insulin reduction assay is commonly used to determine whether a protein can function as an oxidoreductase, regardless of its function in the reducing or the oxidizing pathway in vivo. As shown in Figure 6, HP0231 catalyzes insulin reduction, even more efficiently than EcDsbA.

We also determined the redox potential of HP0231 by equilibrating this protein in a series of redox buffers containing various ratios of reduced and oxidized glutathione (Figure 7). The standard redox potential at $20^{\circ} \mathrm{C}$ calculated for $\mathrm{HP} 0231$ is $-116 \mathrm{mV}$, which is similar to the redox potential of EcDsbA $(-120 \mathrm{mV})[31,32]$.

\section{Discussion}

The E. coli Dsb system is considered to be the paradigm of oxidative protein folding pathways in bacteria. However, bioinformatic analyses of whole-genome sequencing data have revealed that many bacterial disulfide bond-forming systems diverge from the E. coli machinery. It is therefore important to characterize in details the oxidative folding pathways operating in bacteria other than E. coli, including pathogens such as H. pylori. As many virulence factors are indeed stabilized by the formation of one or more disulfide bonds, the characterization of $H$. pylor $i$ Dsb proteins may facilitate a future discovery of an effective anti-Helicobacter drug. A multiparameter selection of $H$. pylori antigens has also marked out HP0231 as a potentially highly protective antigen for vaccine development. Mouse immunization with purified HP0231 indicated its high protective efficacy [33,34]. Immunization of human volunteers with Salmonella Ty2la, the licensed typhoid fever vaccine, producing HP0231 indicated an antigen-specific $\mathrm{CD}^{+} \mathrm{T}$ cell response and mild protective effect [35]. The bioinformatic analysis of $H$. pylori (strain 26695) proteome, conducted with the general localization predictor PSORTb3 [36] and with the meta-predictor MetaLocGramN specialized in predicting subcellular localization for proteins from Gramnegative bacteria [37], indicated that this bacterium has around 150 non-cytoplasmic proteins, containing at least two cysteine residues. Thus, these proteins are potential targets of the $H$. pylori Dsb system (J.M. Bujnicki and M. Magnus, International Institute of Molecular and Cell Biology in Warsaw, personal communication). The list of proteins that potentially form disulfide bonds includes virulence factors such as the vacuolating cytotoxin VacA or proteins that are components of the type IV secretion apparatus encoded by genes clustered in the Cag pathogenicity island. Effectors molecules delivered by this secretion system into eukaryotic cells are involved in interactions with many of the host signal-transduction pathways. Previously, we also demonstrated that a H. pylori mutant impaired in disulfide bond formation exhibits a greatly reduced ability to colonize mice gastric mucosa [9]. 
A

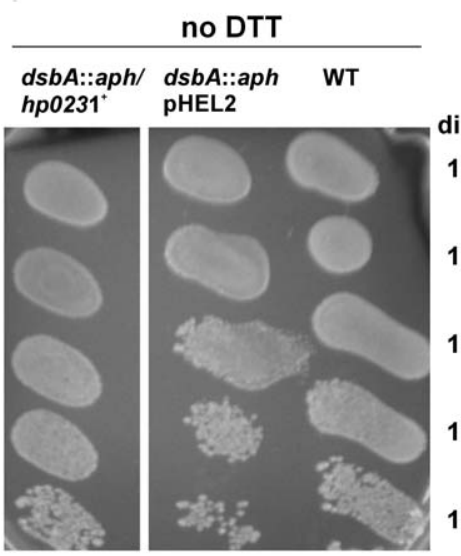

B

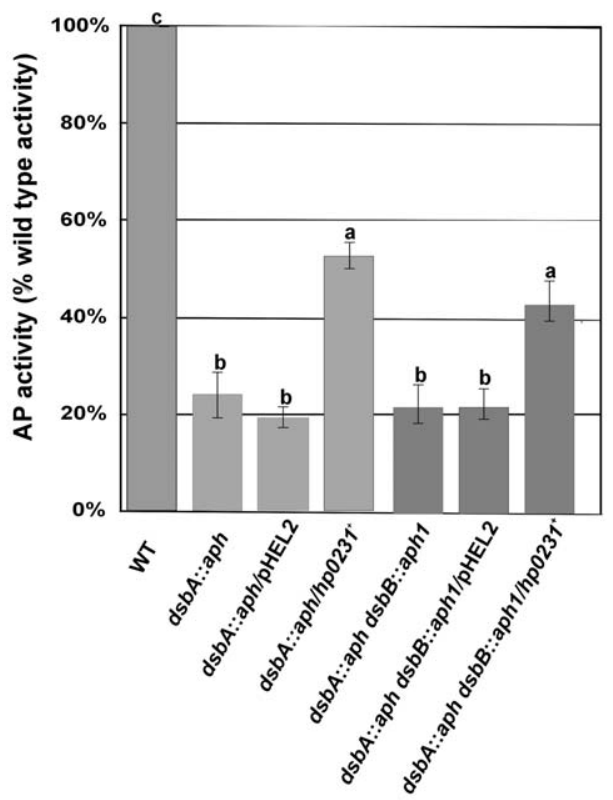

$12 \mathrm{mM}$ DTT

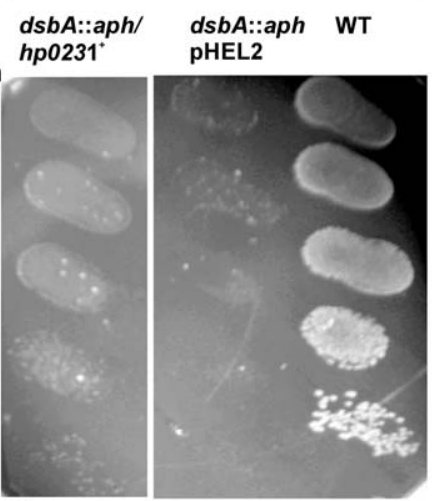

C

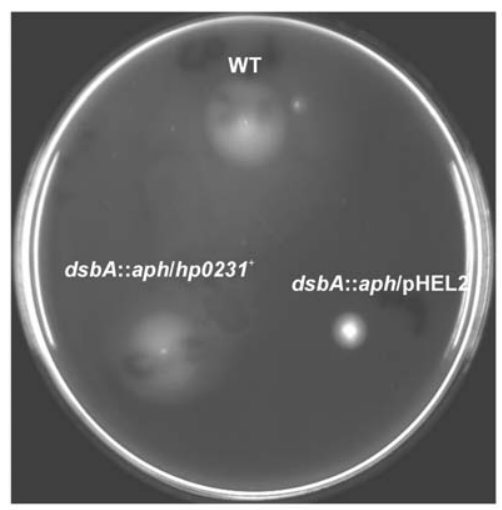

Figure 5. HP0231 restores the $E$. coli $d s b A^{-}$wild type phenotype in three independent functional assays. As a negative control $E$. coli dsbA::aph was transformed with an empty pHEL2 vector. Panel A DTT sensitivity assay; panel B alkaline phosphatase assay. The bars represent average activity of three independent experiments with wild type set to $100 \%$ activity; error bars with the different letters indicate a significant difference $(p<0.01)$ in relative alkaline phosphatase activity between the $H$. pylori $\mathrm{N} 6 \mathrm{wt}$ cells and the mutant strains, and the complementant strains (in ANOVA followed by post hoc Tukey's test). Alkaline phosphatase activity of wild type and $d s b$ mutants and complementants strains was performed in M63 minimal media; Panel C motility assay.

doi:10.1371/journal.pone.0046563.g005

Here, we report the functional and biochemical characterization of HP0231, a H. pylori protein from the Dsb family. First, we show that HP0231 is maintained oxidized in vivo, similarly to EcDsbA. Our data suggest that the protein that maintains HP0231 oxidized is HpDsbI. We also characterized the phenotype of a hp0231 mutant strain and found that it is reminiscent of the phenotype of E. coli $d s b A$ mutants. Whereas an E. coli $d s b A^{-}$strain exhibits a number of phenotypes, no consistent phenotype has yet been found for the $E$. coli $d s b G^{-}$strain. E. coli lacking the periplasmic disulfide isomerase $d s b C$ are sensitive to the redoxactive metal copper as DsbC is required for rearrangements of copper-catalyzed non-native disulfides $[1,20]$. Expression of the HP0231 in the E. coli $d s b A$ mutant complements the motility defect and the sensitivity to DTT, and partially restores the ability to oxidatively fold alkaline phosphatase. However, expression of HP0231 does not complement the E. coli $d s b C$ mutant. Measuring the redox potential of the protein revealed that it has a high redox potential similarly to E. coli $\mathrm{DsbA}$. Additionally HP0231 is able to catalyze reduction of insulin in the presence of DTT whereas EcDsbG is not active in this assay $[2,38]$.

Altogether, our results indicate that HP0231 functions in the oxidizing pathway involving HpDsbI, and that HP0231 is the functional equivalent of EcDsbA. For that reason, we propose to call this protein $\mathrm{HpDsbA}$.

The structure of HP0231 has been recently solved. Intriguingly, $\mathrm{HpDsbA}$ appears as a $\mathrm{V}$-shape protein, like EcDsbC and EcDsbG, two proteins functioning in reducing pathways. Because of this structural similarity, HP0231 was proposed to be the functional homologue of EcDsbG in H. pylori. Moreover, HP0231 was shown in vitro to be able to reduce HP0518, which is a putative L,Dtranspeptidase presenting a single catalytic cysteine residue $[27,39]$. 


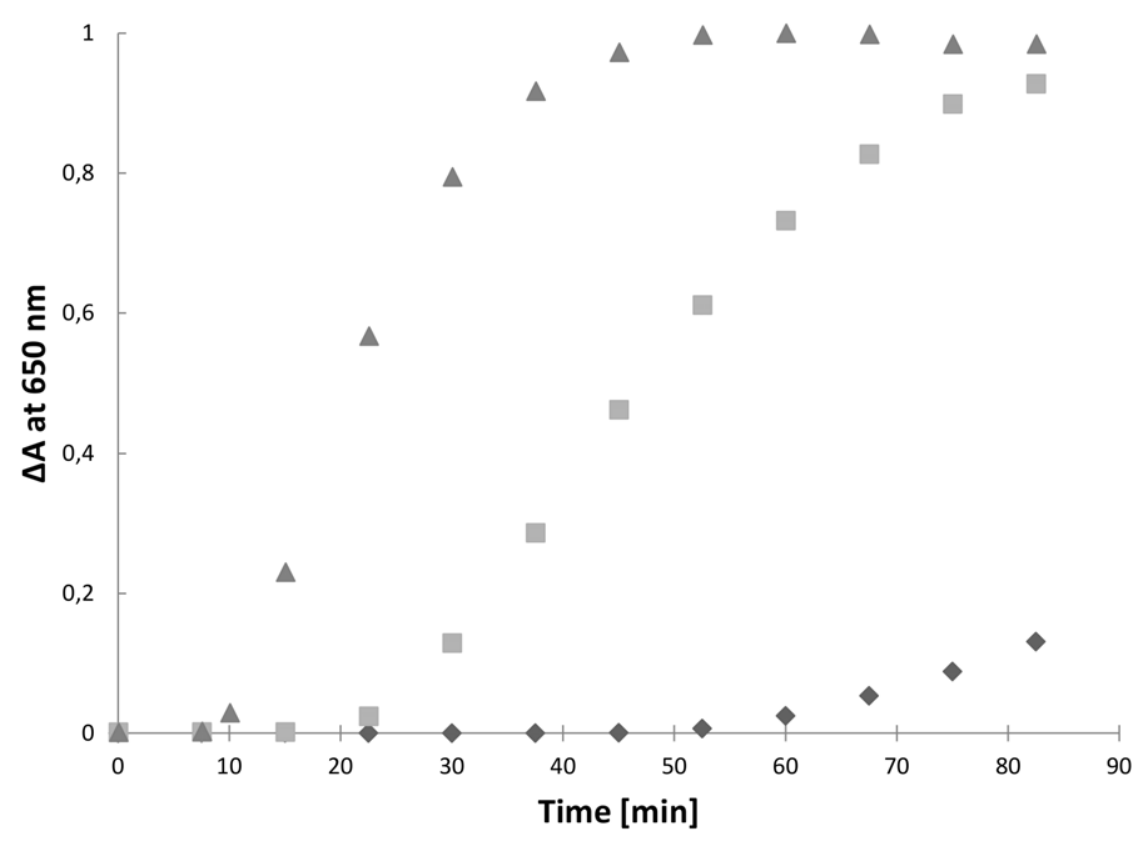

Figure 6. The insulin reduction assay. The reaction mixture contained $150 \mu \mathrm{M}$ insulin in potassium phosphate buffer, pH 7.0 and 2 mM EDTA. The reaction was performed in the absence $(\bullet)$ or presence of $10 \mu \mathrm{M} \mathrm{EcDsbA}(\square), 10 \mu \mathrm{M}$ HP0231 ( $\Delta$ ). Reactions started by adding DTT to the final concentration of $1 \mathrm{mM}$. The changes in the absorbance at $650 \mathrm{~nm}$ as a function of time were measured. Three independent experiments were performed.

doi:10.1371/journal.pone.0046563.g006

These latter data require further clarification as HP0518, according to Asakura et al., exerts its activity in the cell cytoplasm [39]. However, HP0518 has a classical signal sequence and it cannot be excluded that under certain conditions HP0518 maybe secreted [39]. As explained above, our data, and particularly the fact that HP0231 is maintained in an oxidized state in vivo, provide strong arguments against the involvement of this protein in the reducing pathway.

Although individual domains of HP0231 display structural resemblance to the corresponding domains of EcDsbG, there are significant structural variations between the two proteins, which result in different dimer structures. In HP0231, the linker helix is slightly longer as compared to EcDsbG resulting in a different orientation of the $\mathrm{G}$-terminal catalytic domain with respect to the N-terminal dimerization domain [27]. There are also major differences in the amino acid residues lining the $\mathrm{V}$-shaped cleft of HP0231 and EcDsbG. The inner surface of the H. pylor $\mathrm{HP0231}$ cleft is lined with positive residues, which are absent in EcDsbG.

Both the flagellum and the helical shape allow $H$. pylori to penetrate the mucosa and then to colonize and later persist in the host stomach [40]. However, the mechanisms by which H. pylori control its helical shape and cellular motility are not completely

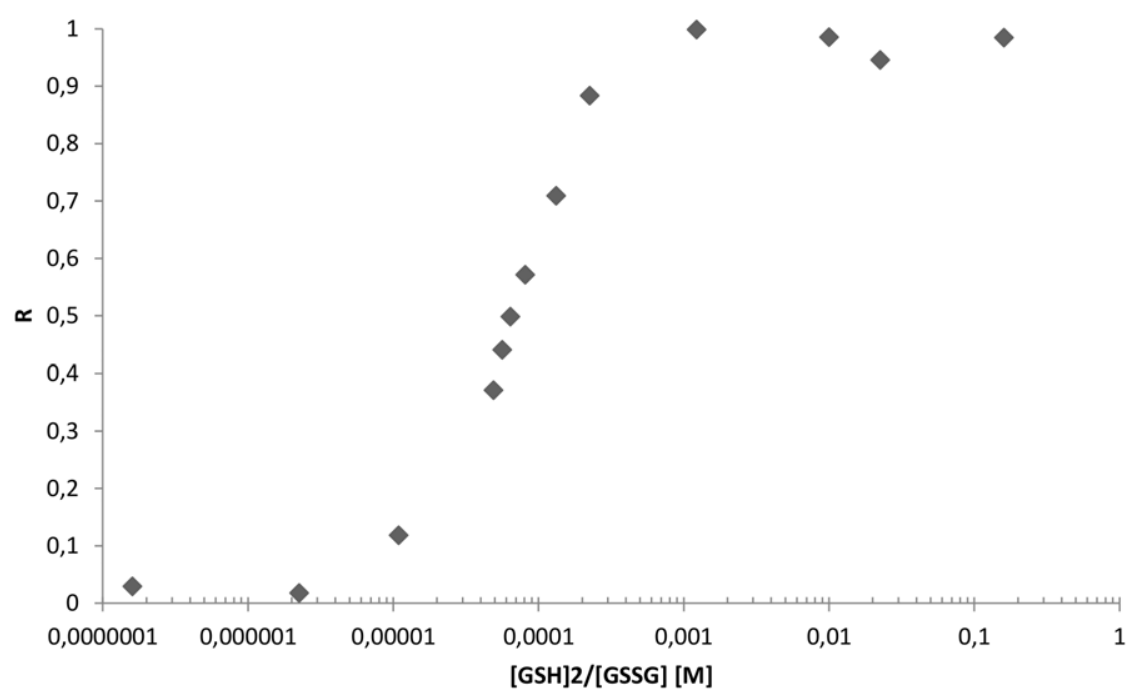

Figure 7. Redox equilibrium of H. pylori HP0231 with glutathione. The fraction of reduced (R) HP0231 was determined using the specific HP0231 fluorescence at $324 \mathrm{~nm}$.

doi:10.1371/journal.pone.0046563.g007 
understood. We showed that the $h p 0231$ inactivation results in cellshape changes and lack of motility, which for now cannot be explained as the cells are normally flagellated. Similarly, Campylobacter jejuni mutants with a defective pflA (paralyzed flagellar protein) gene are also non-motile but possess a flagellum [41,42]. H. pylori HP1274, homolog of PflA, is a predicted extracytoplasmic protein with six cysteine residues. Thus, this protein can be a potential target of HP0231. However this hypothesis requires further clarification.

Additional work will also be needed to understand the role that the Dsb system plays in cell morphology. Remarkably, the shape of H. pylori cells lacking HP0231 resembles that of the H. pylori ccmA (curved cell morphology) mutated strain described by Sycuro et al. [43] and that of the ccrp mutant (HP0059 - one of coiled coil rich proteins) described by Waidner et al. [40].

In conclusion, this study provides new insight into the $H$. pylori disulfide bond formation system, and led to the characterization of the first dimeric oxidoreductase functioning in the oxidizing pathway.

\section{Supporting Information}

Figure S1 Confirmation of $\boldsymbol{h p 0 2 3 1}$ mutation by Westernblot analysis. $H$. pylori wt N6 and N6 hp0231::cat proteins (the whole cell lysate) were separated by $12 \%$ SDS-PAGE and electrotransfered onto a nitrocellulose membrane. Specific rabbit serum with antibodies against HP0231 were used to verify the lack of HP0231 in N6 hp0231::cat mutant cells. The asterisks denote unknown proteins recognized by the antiserum. The relative positions of the molecular weight markers (lanes M) are listed on the left (in kilodaltons).

(TIF)

\section{References}

1. Messens J, Collet JF (2006) Pathways of disulfide bond formation in Escherichia coli. Int J Biochem Cell Biol 38: 1050-1062.

2. Gleiter S, Bardwell JC (2008) Disulfide bond isomerization in prokaryotes. Biochim Biophys Acta 1783: 530-534.

3. Depuydt M, Messens J, Collet JF (2011) How proteins form disulfide bonds. Antioxid Redox Signal 15: 49-66.

4. Kadokura H, Katzen F, Beckwith J (2003) Protein disulfide bond formation in prokaryotes. Annu Rev Biochem 72: 111-135.

5. McCarthy AA, Haebel PW, Torronen A, Rybin V, Baker EN, et al. (2000) Crystal structure of the protein disulfide bond isomerase, DsbC, from Escherichia coli. Nat Struct Biol 7: 196-199.

6. Heras B, Edeling MA, Schirra HJ, Raina S, Martin JL (2004) Crystal structures of the DsbG disulfide isomerase reveal an unstable disulfide. Proc Natl Acad Sci U S A 101: 8876-8881.

7. Depuydt M, Leonard SE, Vertommen D, Denoncin K, Morsomme P, et al. (2009) A periplasmic reducing system protects single cysteine residues from oxidation. Science 326: 1109-1111.

8. Katzen F, Beckwith J (2000) Transmembrane electron transfer by the membrane protein DsbD occurs via a disulfide bond cascade. Cell 103: 769-779.

9. Godlewska R, Dzwonek A, Mikula M, Ostrowski J, Pawlowski M, et al. (2006) Helicobacter pylori protein oxidation influences the colonization process. Int J Med Microbiol 296: 321-324.

10. Raczko AM, Bujnicki JM, Pawlowski M, Godlewska R, Lewandowska M, et al. (2005) Characterization of new DsbB-like thiol-oxidoreductases of Campylobacter jejuni and Helicobacter pylori and classification of the DsbB family based on phylogenomic, structural and functional criteria. Microbiology 151: 219-231.

11. Kaakoush NO, Kovach Z, Mendz GL (2007) Potential role of thiol:disulfide oxidoreductases in the pathogenesis of Helicobacter pylori. FEMS Immunol Med Microbiol 50: 177-183.

12. Suzuki H, Nishizawa T, Tsugawa H, Mogami S, Hibi T (2012) Roles of oxidative stress in stomach disorders. J Clin Biochem Nutr 50: 35-39.

13. Handa O, Naito $Y$, Yoshikawa $T$ (2011) Redox biology and gastric carcinogenesis: the role of Helicobacter pylori. Redox Rep 16: 1-7.

14. Wang G, Alamuri P, Maier RJ (2006) The diverse antioxidant systems of Helicobacter pylori. Mol Microbiol 61: 847-860.

15. Cho SH, Parsonage D, Thurston C, Dutton RJ, Poole LB, et al. (2012) A new family of membrane electron transporters and its substrates, including a new cell envelope peroxiredoxin, reveal a broadened reductive capacity of the oxidative bacterial cell envelope. MBio 3.
Figure S2 RT-PGR analysis of $H$. pylori hp0232 transcription from the wild-type or hp0231::cat mutant chromosomal DNA. Equal amounts of mRNAs isolated from H. pylori cells (wt - lane 1 and hp0231::cat mutant - lane 3) were reverse-transcribed using primer hp232R and the resulting cDNA was PCR-amplified with a pair of primers, hp232F and hp232R. To the control reactions (lanes $2 \& 4$ ) reverse transcriptase was not added. The relative positions of the DNA molecular length markers (lanes $\mathrm{M}$ ) are listed on the left.

(TIF)

Figure S3 HP0231 does not restore the $E$. coli $d s b C^{-}$ wild type phenotype in the copper sensitive assays. As a negative control $E$. coli $d s b C:: a p h$ was transformed with an empty pHEL2 vector. The numbers indicate: 1 - WT, 2 - $d s b C:: k a n, 3-$ $d s b C:: k a n / h p 0231^{+}, 4-d s b C:: k a n / h p 0377,5-d s b C:: k a n / p H E L 2$. (TIF)

\section{Acknowledgments}

We would like to thank prof. J. C. Bardwell, for providing E. coli JCB816, JCB817, JCB818 strains and prof. A. Labigne for providing H. pylori N6 strain. We thank Pawel Laniewski, Janusz Bujnicki and Marcin Magnus for discussions. PR wants to thank JFG lab members for help during the biochemical experiments.

\section{Author Contributions}

Conceived and designed the experiments: PR EKJK JFC. Performed the experiments: PR KAR EW. Analyzed the data: PR EKJK JFC. Contributed reagents/materials/analysis tools: JFG EKJK. Wrote the paper: PR EW JFG EKJK.

16. Sabarth N, Lamer S, Zimny-Arndt U, Jungblut PR, Meyer TF, et al. (2002) Identification of surface proteins of Helicobacter pylori by selective biotinylation, affinity purification, and two-dimensional gel electrophoresis. J Biol Chem 277: 27896-27902.

17. Bumann D, Aksu S, Wendland M, Janek K, Zimny-Arndt U, et al. (2002) Proteome analysis of secreted proteins of the gastric pathogen Helicobacter pylori. Infect Immun 70: 3396-3403.

18. Tomb JF, White O, Kerlavage AR, Clayton RA, Sutton GG, et al. (1997) The complete genome sequence of the gastric pathogen Helicobacter pylori. Nature 388: $539-547$

19. Ferrero RL, Cussac V, Courcoux P, Labigne A (1992) Construction of isogenic urease-negative mutants of Helicobacter pylori by allelic exchange. J Bacteriol 174: 4212-4217.

20. Hiniker A, Collet JF, Bardwell JC (2005) Copper stress causes an in vivo requirement for the Escherichia coli disulfide isomerase DsbC. J Biol Chem 280: 33785-33791.

21. Sambrook J, Russell DW (2001) Molecular cloning: a laboratory manual. Cold Spring Harbor, N.Y.: Cold Spring Harbor Laboratory Press.

22. Zeng X, He LH, Yin Y, Zhang MJ, Zhang JZ (2005) Deletion of cagA gene of Helicobacter pylori by PCR products. World J Gastroenterol 11: 3255-3259.

23. Grabowska AD, Wandel MP, Lasica AM, Nesteruk M, Roszczenko P, et al. (2011) Campylobacter jejuni dsb gene expression is regulated by iron in a Furdependent manner and by a translational coupling mechanism. BMC Microbiol 11: 166 .

24. Studier FW (2005) Protein production by auto-induction in high density shaking cultures. Protein Expr Purif 41: 207-234.

25. Lafaye C, Iwema T, Carpentier P, Jullian-Binard C, Kroll JS, et al. (2009) Biochemical and structural study of the homologues of the thiol-disulfide oxidoreductase DsbA in Neisseria meningitidis. J Mol Biol 392: 952-966.

26. Sardesai AA, Genevaux P, Schwager F, Ang D, Georgopoulos C (2003) The OmpL porin does not modulate redox potential in the periplasmic space of Escherichia coli. EMBO J 22: 1461-1466.

27. Yoon JY, Kim J, Lee SJ, Kim HS, Im HN, et al. (2011) Structural and functional characterization of Helicobacter pylori DsbG. FEBS Lett 585: 3862-3867.

28. Heras B, Shouldice SR, Totsika M, Scanlon MJ, Schembri MA, et al. (2009) DSB proteins and bacterial pathogenicity. Nat Rev Microbiol 7: 215-225.

29. Holmgren A (1979) Thioredoxin catalyzes the reduction of insulin disulfides by dithiothreitol and dihydrolipoamide. J Biol Chem 254: 9627-9632. 
30. Collet JF, D'Souza JC, Jakob U, Bardwell JC (2003) Thioredoxin 2, an oxidative stress-induced protein, contains a high affinity zinc binding site. J Biol Chem 278: 45325-45332.

31. Zapun A, Bardwell JC, Creighton TE (1993) The reactive and destabilizing disulfide bond of DsbA, a protein required for protein disulfide bond formation in vivo. Biochemistry 32: 5083-5092.

32. Inaba K, Ito K (2002) Paradoxical redox properties of DsbB and DsbA in the protein disulfide-introducing reaction cascade. EMBO J 21: 2646-2654.

33. Bumann D, Jungblut PR, Meyer TF (2004) Helicobacter pylori vaccine development based on combined subproteome analysis. Proteomics 4: 28432848.

34. Sabarth N, Hurwitz R, Meyer TF, Bumann D (2002) Multiparameter selection of Helicobacter pylori antigens identifies two novel antigens with high protective efficacy. Infect Immun 70: 6499-6503.

35. Aebischer T, Bumann D, Epple HJ, Metzger W, Schneider T, et al. (2008) Correlation of $\mathrm{T}$ cell response and bacterial clearance in human volunteers challenged with Helicobacter pylori revealed by randomised controlled vaccination with Ty21a-based Salmonella vaccines. Gut 57: 1065-1072.

36. Yu NY, Wagner JR, Laird MR, Melli G, Rey S, et al. (2010) PSORTb 3.0: improved protein subcellular localization prediction with refined localization subcategories and predictive capabilities for all prokaryotes. Bioinformatics 26: 1608-1615.

37. Magnus M, Pawlowski M, Bujnicki JM (2012) MetaLocGramN: A metapredictor of protein subcellular localization for Gram-negative bacteria. Biochim Biophys Acta.
38. Bessette PH, Cotto JJ, Gilbert HF, Georgiou G (1999) In vivo and in vitro function of the Escherichia coli periplasmic cysteine oxidoreductase DsbG. J Biol Chem 274: 7784-7792.

39. Asakura H, Churin Y, Bauer B, Boettcher JP, Bartfeld S, et al. (2010) Helicobacter pylori HP0518 affects flagellin glycosylation to alter bacterial motility. Mol Microbiol 78: 1130-1144.

40. Waidner B, Specht M, Dempwolff F, Haeberer K, Schaetzle S, et al. (2009) A novel system of cytoskeletal elements in the human pathogen Helicobacter pylori. PLoS Pathog 5: e1000669.

41. Yao R, Burr DH, Doig P, Trust TJ, Niu H, et al. (1994) Isolation of motile and non-motile insertional mutants of Campylobacter jejuni: the role of motility in adherence and invasion of eukaryotic cells. Mol Microbiol 14: 883-893.

42. Bleumink-Pluym NM, Verschoor F, Gaastra W, van der Zeijst BA, Fry BN (1999) A novel approach for the construction of a Campylobacter mutant library. Microbiology 145 (Pt 8): 2145-2151.

43. Sycuro LK, Pincus Z, Gutierrez KD, Biboy J, Stern CA, et al. (2010) Peptidoglycan crosslinking relaxation promotes Helicobacter pylori's helical shape and stomach colonization. Cell 141: 822-833.

44. Bardwell JC, McGovern K, Beckwith J (1991) Identification of a protein required for disulfide bond formation in vivo. Cell 67: 581-589.

45. Heuermann D, Haas R (1998) A stable shuttle vector system for efficient genetic complementation of Helicobacter pylori strains by transformation and conjugation. Mol Gen Genet 257: 519-528. 